ÁNGEL JOSÉ MACÍN

\title{
EL SUFRIMIENTO DEL MALVADO EN Sal 32,10
}

\section{Introducción}

Con mucha frecuencia, en el Salterio se hace referencia a los malvados. Por lo general, esta categoría literaria se construye en oposición al salmista, que experimenta la persecución y el acoso de quienes buscan su destrucción. En Sal 17,8-9 ora el salmista diciendo: «Guárdame como a las niñas de tus ojos, protégeme a la sombra de tus alas, de los malvados que me acosan, enemigos que me cercan con saña» ${ }^{1}$.

La figura de los malvados es descripta con bastante amplitud: «Su collar es la soberbia y la violencia los cubre como un manto. La maldad le sale por los poros. Su corazón desborda de malos propósitos. Se burlan, hablan con malicia, gritan y son prepotentes...» (Sal 73,6-8) ${ }^{2}$. En otro pasaje leemos: «El malvado escucha la voz del pecado en el fondo de su corazón. No teme a Dios ni siquiera delante de él, porque se tiene en tan alta estima que es incapaz de reconocer su culpa y detestarla» (Sal 36,2b-3)

1 Las traducciones son mías. En ellas se privilegia especialmente la comprensión de la frase en español, aunque tratando de guardar un respeto fundamental por el tenor literal del texto.

2 Aludo especialmente al uso del término rāšā', aunque se pueden incluir otros vocablos de este campo semántico, como por ejemplo 'āwaen, que podría traducir-

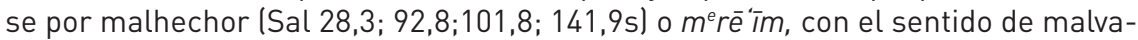
dos (Sal 26,$5 ; 37,9)$. Cito solo algunos de los términos que confluyen en este amplio campo de significación. Por lo general, rāšā se opone a șdyq, con la traducción ha-

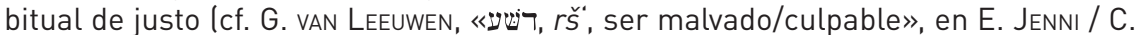
Westermann [eds.], Diccionario teológico manual del Antiguo Testamento II. Madrid, Cristiandad, 1985, cols. 1023-1024).

${ }^{3}$ La lista de Salmos que afrontan la cuestión del malvado en contraposición al justo es extensa. Indico solamente algunos, como punto de referencia: Sal 1; 10; $11 ; 27$; etc. 
No obstante esta situación de seguridad que transparenta la descripción de los malvados, su futuro se estima sombrío, según la tendencia general del Salterio. Así, leemos en Sal 1,4: «Pero no sucederá así con los malvados, serán como paja que se lleva el viento». Una perspectiva similar se percibe en Sal 35 o en Sal 71. En las circunstancias actuales, y siempre según el Salterio, los malvados se muestran seguros, y hasta prepotentes, pero en el futuro cambiará radicalmente su condición, cuando sea reivindicado el justo.

En Sal 32,10, en cambio, se encuentra una afirmación que parece orientarse en otra perspectiva, o al menos incluir algún matiz diferente, aunque permaneciendo siempre en el mismo campo semántico. Se dice que «los sufrimientos del malvado son muchos» ${ }^{4}$. Aparentemente no se habla de una situación futura o de un cambio de situación en el futuro, cosa que frecuentemente se anticipa a los malvados. Se habla de una situación presente. El malvado, mientras se mantiene en esta condición, sufre, padece. ¿Es este realmente el sentido del versículo? ¿Corresponde colocar en la traducción el verbo en presente o conviene hacerlo en futuro? Si se acepta esta particularidad de la frase, cabe todavía una pregunta: ¿a qué tipo de sufrimiento se refiere el salmista?

En este estudio me propongo indagar en tal cuestión, que considero interesante como expresión existencial de las opciones de vida que los seres humanos asumimos y de las consecuencias del camino que cada uno elige recorrer. Secundariamente, una profundización en el significado del texto puede servir para un replanteo del tema del pecado en un ambiente relativista y poco sensible a la responsabilidad personal en la gestión del mal y, al mismo tiempo, con serias dificultades para erradicar el sufrimiento del corazón. No parece una investigación sin importancia en un tiempo donde se quiere redescubrir, de un modo más profundo, el sentido de la misericordia de Dios.

Para el desarrollo de la misma recorreré el siguiente itinerario: en primer lugar voy a comentar de forma general el Salmo 32, señalando

${ }^{4}$ Las traducciones al español de este versículo son variadas. Señalo tres a modo de ejemplo: la Biblia de América (La Casa de la Biblia. Madrid-SalamancaEstella, PPC - Sígueme - Verbo Divino, $\left.{ }^{18} 1997\right)$ traduce por «muchas son las penas del malvado»; por su parte, la traducción española de la Biblia de Jerusalén (Bilbao, Desclée de Brouwer, 1999) dice: «Copiosas son las penas del malvado». La traducción argentina de La Biblia del Pueblo de Dios (Buenos Aires - Madrid, Fundación Palabra de Vida - San Pablo, 1994) elige la siguiente fórmula: «¡Cuántos son los tormentos del malvado!», enfatizando la frase con los signos de exclamación. Nótese que en estas tres traducciones citadas, sugestivamente el verbo se coloca en presente. 
algunos aportes valiosos de la investigación exegética contemporánea sobre el mismo. Luego voy a tratar de precisar el sentido del v. 10 en el contexto, para poder extraer, posteriormente, algunas conclusiones vinculadas a la teología y a la vida espiritual ${ }^{5}$.

\section{La experiencia del mal y del perdón}

\subsection{Consideraciones generales: género literario, traducción y estructura general de Sal 32}

El texto, en su conjunto, parece expresar una experiencia personal de liberación y salvación. El Sal 32 es el segundo de los denominados «salmos penitenciales» tradicionales $(\text { Sal } 6 ; 38 ; 51 ; 102 ; 130 ; 143)^{6}$. No se trata tanto de una oración para pedir perdón cuanto de un testimonio, de un relato sobre los beneficios de pedir perdón, con una enseñanza ulterior y más amplia, basada en la experiencia personal descripta ${ }^{7}$.

Mirando con cierto detenimiento el encadenamiento de los versos se puede concluir que no conviene fijar un género unitario para todo el salmo. El mismo tiene secciones que corresponden a una especie de liturgia penitencial y otras que tienen una clara construcción sapiencial y didáctica. En todo caso se podría hablar de una plegaria penitencial-didáctica.

${ }^{5}$ Una importante discusión exegética está centrada en la fijación de los límites finales del salmo. Su inicio no genera demasiadas dificultades, ya que presenta un claro cambio de vocabulario y de estilo respecto del salmo anterior. Mucho más complicado es fijar el final del mismo. S. D. Snyman estudia extensamente la cuestión, indicando cuatro posibles opciones: al la división tradicional entre Sal 32 y 33 ; b) conectar el Salmo 32 al salmo siguiente, componiendo un solo y único salmo; c) asumir el v. 11 como inicio del Salmo 33; d) tomar el v. 1 del Salmo 33 como final del Salmo 32. Cada una de estas opciones tiene su fundamentación razonable y sus dificultades Icf. S. D. Snyman, «Psalm 32 - Structure, Genre, Intent and Liturgical Use», en D. J. Human / C. J. A. Vos, Psalms and Liturgy. Londres - Nueva York, T. \& T. Clark International, 2004, pp. 155-158). En el presente trabajo adopto una posición ligeramente distinta, aunque en línea con una de las propuestas enumeradas. En la presentación general sigo la división clásica por una cuestión práctica. Después, a la hora del análisis, opto por el v. 10 como conclusivo y recapitulador del salmo, mientras que el v. 11 sería un texto de transición que prolonga lo desarrollado en los versículos previos, pero que también abre paso al contenido del salmo siguiente.

6 J .L. Mars, Salmi. Turín, Claudiana, 2010, p. 165.

7 Ibid. 
Su estructura parece incluir cuatro momentos principales: una proclamación de la felicidad que acarrea el perdón en forma de bienaventuranza; una alusión a la experiencia personal, que pasa del silencio a la confesión de la culpa; la constatación del cambio de situación, luego de producida la confesión de los pecados; finalmente, la contemplación, con rasgos sapienciales, de las consecuencias que provoca el perdón en el pecador $^{8}$. Tal estructura se puede visualizar mejor en la siguiente segmentación del texto:

\section{I.}

${ }^{1}$ Feliz quien recibe el perdón de su culpa, quedando cubierto su pecado.

${ }^{2}$ Feliz el hombre a quien Yahvé no le imputa el delito y en cuyo interior no hay engaño.

II.

${ }^{3}$ Guardaba silencio y se consumía mi cuerpo cansado de gemir todo el día, ${ }^{4}$ porque día y noche tu mano pesaba sobre mí; mi vida ardía como un campo que sufre el calor del verano. ${ }^{5}$ Pero reconocí mi pecado y no te escondí mi culpa; me dije: «Confesaré a Yahvé mis rebeldías», y tú absolviste mi culpa y perdonaste mi pecado.

III.

${ }^{6}$ Por eso, quien te ama te invoca en el momento de la angustia.

Aunque las aguas caudalosas se desborden,

8 Las discusiones han sido importantes en lo que se refiere a la estructura del salmo y las propuestas, variadas. Para un panorama más amplio sobre la cuestión remito al trabajo de P. AufFRET, «Essai sur la structure littéraire du Psaume XXXII», en VT (1988), pp. 257-285. También algunas consideraciones al respecto en S. D. SNYMAN, «Psalm 32», pp. 159-163. 
jamás le alcanzarán.

${ }^{7}$ Tú eres mi refugio,

me consuelas en la angustia,

me rodeas con tu salvación.

IV.

${ }^{8}$ Voy a instruirte, a mostrarte el camino a seguir;

sin quitarte los ojos de encima seré tu consejero.

${ }^{9}$ No seas como el caballo o la mula,

a los cuales hay que domar con freno y bozal ${ }^{9}$,

si no, no puedes acercarte.

${ }^{10}$ Muchos son los sufrimientos del malvado,

pero a quien confía en el Señor su amor lo protege.

${ }^{11}$ Alégrense, justos, en Yahvé,

canten llenos de gozo los de corazón recto ${ }^{10}$.

En esta segmentación del salmo se nota una mayor cohesión en las tres primeras secciones. La primera funciona claramente como introducción general, con el lenguaje de las bienaventuranzas. Las afirmaciones se hacen en tercera persona y se repiten con expresiones diversas, tratando de indicar la convicción del salmista en este punto. La segunda sección describe dramáticamente la experiencia del pecado, la confesión del mismo, que se produce por lo insostenible de la situación, y el perdón de Dios, que llega como consecuencia de dicha confesión. La alegría y el bienestar que provoca la confesión del pecado se describe en la tercera sección, que va unida estrechamente a la sección antecedente. Para la cuarta sección se dejan las consideraciones de tipo sapiencial, las cuales, evidentemente, presentan un carácter más heterogéneo. Se pasa del trato personal con Yahvé a algunas generalizaciones que tienden a extraer conclusiones de lo presentado en las secciones anteriores. De esta forma, el salmo muestra una notable combinación entre experiencia personal y conclusiones de carácter más general, válidas para todos.

9 No son fáciles de traducir los aparejos mencionados en el texto hebreo. Me parece que, más allá de la distancia en el tiempo y las diferencias culturales, estas expresiones se refieren a los instrumentos que sirven para dominar a un animal de monta o de carga.

10 Tal como he indicado en una nota anterior, en la traducción trato de respetar el tenor literal del texto, pero procurando hacerlo accesible a la lengua española. Se incluye en la traducción el v. 11, con las precisiones ya señaladas previamente. 


\subsection{Comentario a cada sección}

Siguiendo este esquema general se pueden realizar algunas observaciones específicas sobre este canto al perdón y la misericordia de Yahvé. En la primera parte se encuentran dos bienaventuranzas que funcionan casi en forma paralela, aunque el lenguaje utilizado es diverso ${ }^{11}$.

I. Llama la atención la suma de expresiones para designar el pecado o la transgresión: peša, hața' y 'awôn. La primera se pensó inicialmente vinculada a la rebelión. Después de un minucioso análisis de la etimología del vocablo, R. Knierim llega a la conclusión de que se trata «más bien de un concepto genérico formal que sintetiza en sí las distintas clases de delitos contra personas y cosas» ${ }^{12}$. Las restantes son expresiones frecuentes para indicar la realidad del pecado como transgresión, como distorsión en el camino del bien. La expresión 'awôn parte del significado básico de torcer, doblegar, mientras que hața' significa errar el tiro, no dar en el blanco. De esta manera se plantea ya desde el comienzo una notable amplitud en lo que se refiere a la identificación del sujeto que se encuentra en una situación comprometida delante de Dios. Incluso la fórmula final berûhô remiyya $\bar{a}$ se ubica en esta misma línea, describiendo una situación irregular pero desde una formulación positiva. Se valora, en este caso, la inexistencia de doblez o de intención equivocada en el interior de una persona.

Los verbos que van asociados a estas situaciones de pecado son típicos del AT para describir esta temática: se habla de cubrir el pecado y no imputarlo, no tenerlo en cuenta. Se trata del verbo naśa', que se suele traducir por disculpar, olvidar, y del verbo kasah, con el sentido de cubrir. Dos modalidades que todavía se refieren a un aspecto externo del perdón, aunque el sentido del conjunto del salmo intente darle más profundidad al tema y hurgar en las vivencias más interiores del pecador ${ }^{13}$.

11 Un cierto paralelismo sinonímico se nota a nivel estilístico en esta primera parte del salmo. La construcción literaria es rica en vocabulario.

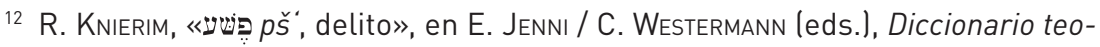
lógico manual del Antiguo Testamento II, cols. 616ss.

13 Se ha discutido sobre el tema de la justificación a propósito del uso de estos verbos. Señala R. E Murphy al respecto: «Estos versículos no han de leerse a la luz de la teología escolástica o de los reformadores; en ellos no se dice nada sobre la naturaleza intrínseca de la justificación. Las expresiones "cubrir" e "imputar" son descripciones antropomórficas del perdón de Dios y aparecen con frecuencia en el AT» (R. E. Murphy, «Salmos», en R. E. Brown / J. A. Fitzmyer / R. E. Murphy (eds.), Comentario bíblico San Jerónimo II. Madrid, Cristiandad, 1971, p. 626). 
La felicidad está definitivamente del lado del justo ${ }^{14}$. No son compatibles la felicidad y una vida de pecado. Una felicidad que parece estar planteada para la situación presente. No se está refiriendo a una situación futura. El justo ha sido objeto de la remisión de sus culpas, puede experimentar la felicidad y la alegría. Se trata de una constatación que tendrá su sustento en los versículos que siguen a continuación, la cual pasa por la confesión del pecado.

En el centro de todo el segmento se encuentra el nombre de Yahvé, quien claramente es el sujeto que ofrece el perdón al pecador arrepentido. Este aspecto es uno de los aportes más hondos de todo el AT, que ve en Yahvé al único capaz de perdonar los pecados. La felicidad, por otra parte, brota de esta comunión con Yahvé, quien ofrece el perdón al pecador arrepentido.

II. La segunda parte, que constituye el corazón del contenido de esta pieza literaria, encierra elementos interesantes para comprender la psicología del pecador desde la perspectiva del salmista y su relación con la culpa. Al planteo más general, observado en los primeros versículos, sigue esta descripción más interna de la experiencia del pecador.

Sobresale la mención del silencio en su aspecto negativo y destructor $^{15}$. El silencio respecto del pecado conduce al pecador a consumirse en vida. Hay una oposición entre el silencio y el hablar. En cierto sentido, la actitud de callar recuerda el texto de Gn 3,8, en el cual Adán se esconde de la presencia de Dios, luego de haber transgredido el mandamiento que le había sido impuesto. El silencio sería, entonces, la reacción típica del pecador, del transgresor, quien tiende a ocultar y a esconder su estado irregular. En otros términos, sería una expresión de la ruptura de la confianza con un Dios cercano.

La situación cambia con la confesión del pecado, que en este caso se hace directamente a Yahveh ${ }^{16}$. No parecen encontrarse mediaciones

14 Esto será retomado de una manera más amplia y contundente en el v. 11, que funciona a modo de inclusión del conjunto.

15 En su estudio sobre el silencio en la primera parte del Salterio, E. Ruiz señala este carácter negativo del silencio en Sal 32: «El silencio en el Sal 32 no tiene, pues, un sentido positivo. Se trata del silencio respecto del pecado y se entiende como una actitud de cerrazón frente a Dios, de negarse a confiar en él, que produce sufrimiento» (cf. E. R. RuIz, «El silencio en el primer libro del Salterio [Salmos 1-41]», en RevBib 67 [2005], p. 62).

${ }^{16}$ Hay un contraste entre el «callarse» y «confesar» que resulta funcional a la idea que el salmista quiere ofrecer a los orantes (cf. J. L. MAYs, Salmi, p. 167). 
concretas como marco de dicha confesión ${ }^{17}$. La misma es aquí el comienzo de una vida nueva, de una situación diferente y radicalmente distinta para el pecador. La confesión del pecado y el perdón que Yahvé ofrece producen un cambio decisivo en la vida del pecador, quien vive esta situación como experiencia de liberación y de alivio interior.

Las metáforas que ilustran estas circunstancias son potentes y dejan en claro que el pecado acaba por destruir la vida. Concretamente, la metáfora que describe la experiencia de quien todavía se encuentra en una situación de pecado habla de consumirse, de secarse, de extinguirse, en la estructura física fundamental de una persona. Esto llega al soporte principal del ser humano como el esqueleto, los huesos, que dan consistencia al cuerpo humano. La expresión usada es 'eșem, que se puede traducir básicamente por huesos (cf. Gn 2,23; Nm 19,18), pero que también indica, con mayor amplitud, toda la interioridad del ser humano, como sucede en $\mathrm{Jr}$ 20,9, donde se habla de fuego abrasador que consume, comenzando por los huesos, el conjunto de la estructura corporal. De un lenguaje que parte de una observación de la naturaleza, en este caso humana, se pasa al plano existencial. El consumirse de los huesos indica que el pecado quiebra la estabilidad y la consistencia del ser humano, afectando a su sustancia.

El v. 4 prolonga con otras imágenes esta situación del pecador. En el primer verso se utiliza la imagen de la «mano de Yahvé», la cual ejerce su presión sobre algo y lo oprime, en este caso el pecador. Se trata de un antropomorfismo significativo que indica cierta permanencia o continuidad del vínculo entre el pecador y Yahvé, a pesar de la ruptura que produce el pecado. Ese vínculo se da por la experiencia de la culpa. El tema no es sencillo. Afirma H. W. Jüngling, comentando este versículo: «Dios no acosa al hombre, sino que hace que el hombre llegue ante todo a él y le concede graciosamente una existencia jubilosa ${ }^{18}$. La segunda parte es una expre-

17 Evidentemente, el texto no podría estar refiriéndose a la confesión sacramental tal cual la conocemos hoy en la Iglesia. La confesión indicada en este salmo tiene que ver con la extroversión, con la expresión verbal de aquello que oprime interiormente al pecador.

18 H. W. Jüngling, «Salmos 1-41», en W. R. Farmer / A. J. LeVoratt। y otros (eds.), Comentario Bíblico Internacional. Estella, Verbo Divino, 1999, p. 741. Inmediatamente agrega: «El ojo de Dios sobre el hombre no es el ojo de quien vigila al hombre lcf. Job 7,18-20), sino que es la presencia de Dios, una presencia liberadora y que hace que él llegue a encontrarse a sí mismo» (ibid.). Para tener un panorama más claro sobre esta temática habría que profundizar en la cuestión del «castigo de Dios», algo que nos conduciría a desviarnos, en cierto modo, de la presente investigación. Prefiero, entonces, mantenerme en una posición más existencial, atendiendo al itinerario del salmista y sus conclusiones sobre la experiencia humana. 
sión muy particular que se refiere a alguna cosa que pierde consistencia por el calor. El texto paralelo de Nm 11,8 es por demás elocuente, ya que en esa circunstancia se está describiendo el maná, alimento que es concebido como una especie de pastel ${ }^{19}$. La traducción es difícil, y más todavía el sentido de la frase. Yo propondría hablar de «mi consistencia se desvanecía» o, en un lenguaje más llano, «se desmoronaba toda mi vida». De todas maneras, el carácter sintético de la metáfora hebrea es casi imposible de traducir de un modo preciso. De esta manera, el v. 4 complementa, quizá desde un punto de vista más externo y objetivo, lo dicho en el v. 3. Hay una especie de mutua iluminación en el contenido de ambos versículos.

El v. 5, finalmente, cierra la sesión, señalando el movimiento que va desde la confesión del pecado al perdón de Yahvé ${ }^{20}$ y el resultado de dicho movimiento, que consiste en el perdón de la culpa correspondiente al pecado. La característica más notable del versículo es el énfasis en la confesión, dicho de dos modos diferentes, y el subrayado del perdón, que aparece con una fórmula de carácter intensiva. La confesión, aunque realizada en un marco individual, directamente con Dios, tiene el tono solemne de una celebración litúrgica ${ }^{21}$.

III. La tercera parte adopta el lenguaje didáctico-sapiencial para extraer las consecuencias de lo señalado anteriormente. No tiene la cohesión literaria de las secciones anteriores. El cambio de persona es llamativo entre los vv. 6 y 7. En este último versículo, el tono es más confidencial y está expresado en segunda persona, a modo de plegaria directamente dirigida a Yahvé.

La sección se inicia con una indicación que resume lo dicho anteriormente. Según el v. 6, el amor es lo que mueve al pecador a dirigirse, a remitirse nuevamente a Yahvé, que es el único capaz de salvarlo. La fórmula que aconseja «buscar al Señor mientras pueda ser encontrado» es típica del lenguaje veterotestamentario (Is 55,6; Dt 4,7). La figura de la inundación refuerza la insistencia en esta urgencia de buscar a Yahvé. La inundación,

19 Aunque no se pueda llegar a precisar el punto de partida de esta comparación, es evidente que la expresión lasad se refiere a una comida o algo parecido que tiene una estructura endeble y que puede derretirse fácilmente con el calor. La traducción inglesa cake es significativa para la comprensión de este punto.

${ }^{20}$ Nuevamente se utiliza el verbo nasa' con un sentido parecido al que he indicado oportunamente. En el uso del vocabulario se percibe la cohesión de la primera parte del salmo.

${ }^{21}$ Insisto en la necesidad de aclarar que no estamos en tiempos de la confesión sacramental. El contexto nos lleva a pensar en otro marco. Quizá la liturgia penitencial del Templo o de la sinagoga, de acuerdo a la datación que demos a este texto, puede ser el Sitz im Leben más apropiado para comprender esta confesión. 
como desgracia real o metafórica, es conocida sobre todo en textos proféticos y salmos. Merecen especial mención Is 28,2.15.17-18; Ez 13,11.13; Sal 69,3.16; 124,422. Decir que Dios protege de la inundación significa afirmar que Dios protege del mal a quien confía en él. La inundación también puede ir asociada a la muerte y a la destrucción de la vida de una persona.

En el v. 7 aparece uno de los motivos principales en los que insiste el salmo. Se habla ahora de experiencia de la angustia. De algún modo, lo dicho anteriormente sobre la inundación se expresa con un lenguaje más técnico. Efectivamente, la angustia podría ser descripta como una experiencia de ahogo, de opresión, de falta de respiración. Todo esto se ve vinculado a la imagen de la inundación ${ }^{23}$. El tono es aquí realista, señalando que Dios es el refugio en momentos de angustia y desesperación. Es interesante notar la acumulación de acciones que produce el texto en cuanto a la acción de Dios en favor de quien reconoce su culpa y su pecado. En realidad, hay una suma de acciones de parte de Yahvé y otro tanto de parte del pecador, que otorgan gran movilidad a la pieza literaria.

Los vv. 8-9 son claramente una instrucción sapiencial, introducida por expresiones como «voy a instruirte», «voy a mostrarte el camino a seguir», típicas de la tradición sapiencial (Job 15,17; Prov 1,1ss). Incluso la denominación «consejero» para indicar quien está comunicando esta instrucción tiene un marcado tono sapiencial, aunque su sentido es más polémico (cf. Sir 37,7-8). El sujeto es el sabio que ofrece la instrucción, aunque algunos piensan que puede ser el mismo Yahvé quien está hablando. Estas apreciaciones no necesariamente se excluyen, ya que Yahvé puede ser presentado como sabio y, al mismo tiempo, puede aludir a la figura del sabio humano.

22 Cf. L. Alonso Schökel / C. Carniti, Salmos I. Estella, Verbo Divino, 2008, p. 500.

${ }^{23}$ Es muy importante tener en cuenta el carácter concreto de la lengua hebrea y su notable riqueza semántica. La figura de la inundación sirve para indicar la desesperación de quien experimenta la impotencia frente a una situación insuperable. Algo parecido sucede con las avispas, las cuales aparecen en algunos textos con un sentido básico para indicar el pequeño insecto, pero también son utilizadas para indicar la experiencia del pánico que produce la agresión de un enjambre de avispas o de un ejército. Cf., por ejemplo, los textos de Dt 7,20, donde el sentido de la palabra șiréah alude elípticamente al pánico que padecieron los pueblos que se enfrentaron con Israel, mientras que en Ex 23,28 y Jos 24,12 el vocablo parece referirse metafóricamente al animal concreto o a algún tipo de plaga que Dios envió contra estos pueblos. L. H. Rivas describe con precisión el vínculo entre el insecto y el pánico que el mismo produce, inscribiéndose así el término en el lenguaje metafórico. Aclara, sin embargo, el exegeta argentino que algunos autores prefieren traducir directamente la expresión șireảh como desánimo, depresión, con lo cual desaparece la metáfora (cf. L. H. RIvAs, Diccionario de símbolos y figuras de la Biblia. Buenos Aires, Amico, 2012, pp. 27-28). 
La metáfora de los animales que aparece en el v. 9 tiene su complejidad. Normalmente es interpretada como un llamado irónico a seguir la instrucción sapiencial y a no imitar al caballo y la mula, a los que solamente se puede dominar con aparejos de fuerza. G. Castellino ha propuesto una traducción que sostiene esta perspectiva. Los caballos y las mulas «no prestan atención al freno y la brida», por lo tanto expresan simbólicamente la actitud de los necios ${ }^{24}$. Si se acepta esta opción, la expresión mak'ôbim podría ser entendida como «azotes» que aplica Yahvé al malvado para que cambie de conducta ${ }^{25}$. Esta lectura es interesante en su tenor literal, aunque no resulta tan sencillo sostener la traducción propuesta y su sentido para el conjunto del versículo y del salmo. En todo caso, se puede pensar en una interpretación más sencilla, que consiste en la racionalidad de buscar el camino de la confesión y del perdón de la culpa para evitar el sufrimiento que conlleva una vida signada por el pecado. No animarse a reconocer el pecado y a confiar en el perdón de Dios se constituye, así, en una actitud irracional que acercaría al ser humano al modo de proceder de los animales. Incluso puede leerse de fondo una apelación a la libertad humana, que puede aceptar el perdón sin una imposición por parte de Dios.

Llegados a este punto hay que considerar el v. 10, el cual podría ser leído en esta línea, como una consecuencia de tipo sapiencial que refuerza lo anterior o lo dicho hasta el momento, o, en cambio, ser considerado como un momento diferente en la secuencia del salmo, donde se hace una especie de recapitulación de todo lo expuesto. Si se considera que el v. 11 ya es un versículo de transición que conecta esta unidad literaria con la que sigue es más factible aceptar el carácter recapitulador del v. 10 y vincularlo estrechamente a lo que hemos comentado hasta aquí. Me inclino por esta posición para las observaciones siguientes.

\section{Los sufrimientos del malvado son muchos}

Llegando al punto fundamental de la investigación, fijo la atención en la cuestión del sufrimiento del malvado y sus características, según el tenor del texto. Si se siguiera una perspectiva etimológica, estudiando el uso de la expresión «malvado» rāšă ', no habría demasiados comentarios que hacer. Si sumamos otros datos a este análisis, entonces la cosa puede resultar diferente.

24 Cf. G. Castellino, «Psalm XXXII 9», en VT 2 (1952), pp. 40-41.

${ }^{25}$ Cf. E. R. RuIz, «El silencio en el primer libro del Salterio», p. 61. 
En el Salterio, los malvados son mencionados 82 veces, la mayor parte para indicar a los enemigos de los justos. Algunas veces se asocian a los pueblos extranjeros y son ubicados en paralelismo con los enemigos, hacedores del mal, mentirosos ${ }^{26}$. Es muy interesante la indicación que trae H. Ringgren al respecto, afirmando que en el desarrollo semántico de esta palabra está de fondo la idea de la persona que no está en el lugar propio, en el camino justo, que no cumple la función prevista ${ }^{27}$.

En numerosas oportunidades son descriptos como arrogantes (Sal 10,3-11), desprecian a Yahvé, piensan que no existe Dios. Creen no vacilar jamás. Su boca está llena de falsedad y violencia ${ }^{28}$. Su suerte cambiará cuando actúe Yahvé, de acuerdo a lo afirmado en otros salmos.

Si se tomara en consideración esta orientación, la traducción de nuestro versículo debiera contener el verbo en futuro, afirmando después de la intervención de Yahvé que los malvados sufrirán muchas penas. El uso de la expresión haría pensar en una alusión típica a la oposición justos-malvados, con la descripción correspondiente de la situación actual y el cambio de situación en el futuro por intervención de Dios.

Sin embargo, creo que, si se observa con atención la frase en la que se encuentra esta expresión y el conjunto de Sal 32, algún matiz diferente se puede percibir y apuntar.

El conjunto de la frase del v. 10 es una oración adversativa, que construye su sentido uniendo dos situaciones diferentes. La primera parte de la expresión es una frase nominal compleja, rabbim mak'ôbim lárásá', compuesta por adjetivo + sustantivo + adjetivo sustantivado, lo cual complica la traducción. Así lo hace notar B. Wilmes, señalando que la frase no permite inferir claramente su aspecto temporal ${ }^{29}$. El verbo está implícito, lo cual hace más complicada una traducción precisa y definitiva. Es evidente que, para proponer el verbo en su estado más adecuado, hay que recurrir al contexto literario próximo, esto es, a la segunda parte de la adversativa y, secundariamente, a frases que puedan funcionar en paralelo. La segunda parte de la frase tiene el verbo en imperfecto, lo cual podría autorizar una traducción en futuro, del tipo «pero para quien mantenga su confianza en el

${ }^{26}$ Cf. H. RINGgren, «rāšā"», en GLAT VIII, p. 623.

27 Ibid.

28 Ibid.

29 Concretamente señala el autor: «[...] v. 10a ein Nominalsatz ist, lässt sich das Tempus nicht eindeutig bestimmen» (B. WILLMES, Freude über die Vergebung der Sünden: Synchrone und diachrone Analyse von Salm 32. Frankfurt, Josef Knecht, 1996, P. 78), tratando de deducir el posible sentido de la expresión a partir de otros textos del TM. 
Señor, la misericordia lo rodeará». Sin embargo, no parece que esta sea la temporalidad más aconsejable, cuando en estos versículos finales del salmo se extraen las consecuencias de una experiencia de silencio y posterior confesión que produce un cambio en la existencia del pecador. La confianza ya es una cosa constatada por el salmista. En esta sección de tipo sapiencial se están indicando las consecuencias concretas y tangibles de la confesión de la culpa. Eso reclama el verbo en presente o, mejor, en una función que indique la acción recibida, ya cerrada. De ahí que la mayoría de los traductores opten por una formulación en presente, a modo de proverbio o de apotegma: «Pero al que confía en el Señor, la misericordia lo rodea».

Si se observa el contexto más amplio, se puede inferir que la consideración sapiencial del v. 10 se corresponde con el doble movimiento de callar en un primer momento y confesar la culpa luego. La experiencia del pecador se proyecta así sobre la figura del malvado, ayudando a comprender que quien se encuentra en una situación de pecado sufre y se consume en vida. No se está planteando una hipótesis, sino una constatación, que, por lo tanto, se tiene que expresar en el lenguaje correspondiente. De esta manera, tanto la construcción de la frase como el contexto próximo de la primera parte del v. 10 autorizan a una traducción en presente.

Resumiendo, se puede decir que, si se adopta una mirada amplia respecto del término y su etimología, no hay mucho por agregar respecto de la perspectiva habitual del Salterio en cuanto a la figura del malvado. Sin embargo, si se observa el contenido, el contexto inmediato, es decir, el contenido del Salmo 32, el encadenamiento de las frases y su sentido, la traducción de la frase nominal en cuestión podría incluir una perspectiva un tanto diferente, con un tiempo verbal distinto. Si lo que se toma como referencia es el sufrimiento padecido por el justo mientras se mantenía en el pecado, la expresión podría incluir un matiz referido al presente. Se establecería, de este modo, una peculiar solidaridad entre el pecador y los malvados que permanecen en el pecado o, mejor, un contacto existencial que abre la puerta a un potencial diálogo salvífico que transforme la existencia del malvado, o al menos una mirada compasiva, distinta, sobre la misma. En otras palabras, el paso por la experiencia de disolución realizada por el pecador le lleva a adoptar una mirada más «comprensiva» sobre la vida de quien permanece en una situación de pecado, por más que se mantenga la categorización de «malvado».

Pienso que la observación del contexto literario cercano puede complementarse con la construcción completa de la frase, que elogia al justo que confía en el Señor, cuya acción es ya presente. De esta manera, aunque no todas las dudas quedan despejadas y las potenciales variantes de traducción persistan, se puede afirmar que la inclusión del verbo en presente en la frase nominal 
previamente citada es legítima y condice con el contexto literario inmediato ${ }^{30}$. Se establece, de este modo, una conexión entre la experiencia del pecador arrepentido y perdonado con la figura del malvado. Una conexión que viene dada, fundamentalmente, por cierta solidaridad en la experiencia que se vive cuando uno se encuentra en el camino errado o en la senda equivocada.

\section{El sufrimiento de quien se encuentra en el camino equivocado}

Si lo que he podido argumentar en el apartado anterior respecto de la traducción al presente del verbo implícito en la frase nominal «muchos son los sufrimientos del malvado» es aceptable, entonces se pueden derivar de esta lectura e interpretación del versículo algunas consecuencias teológicoespirituales relevantes para la vida de las personas, en particular en el tiempo que nos toca transitar.

Es una realidad bastante fácil de constatar que a nuestra generación le cuesta reconocer el sentido del pecado, y más todavía sostener que quien se encuentra en una situación de pecado padece sufrimientos y penas. Como pasa con frecuencia en los salmos, la opinión generalizada es que quien elige un camino equivocado disfruta de la situación, aunque posteriormente tenga que aceptar las consecuencias. La tensión presente-futuro suele predominar en los análisis y comentarios sobre este tipo de situaciones. Si se asume la lectura del salmo en una perspectiva diferente, es decir, como una descripción del presente, de la existencia actual del malvado, entonces la cuestión es otra. En el fondo, aunque no lo reconozca o ni siquiera lo perciba, el malvado sufre, padece por su situación, por la posición en la que se encuentra, por haber torcido el camino, por no estar en el lugar y en el cauce que le corresponde a su ser más profundo. Sería una situación similar a cuando uno se encuentra en una fiesta sin lograr sintonía con los invitados o sin conocer demasiado a los demás partícipes del encuentro; otro ejemplo podría tomarse de la persona que tiene que soportar el uso de una vestimenta no adecuada a su físico. Ejemplos banales, claro, para significar algo mucho más hondo. Se trata de una incomodidad, de una inadecuación profunda de quien no vive aquello para lo cual fue pensado y proyectado.

Quizá, arriesgando un poco en el comentario, no se trata de un sufrimiento consciente, ni siquiera reconocido como tal. Pero el fondo de su

30 Ya he señalado al inicio de este estudio la tendencia de los traductores a incluir el verbo en presente para esta construcción nominal. 
alma no puede dejar de contener el malestar que implica encontrarse fuera del rumbo conveniente, ajeno a su condición más propia y esencial.

Si esto es así, la primera consecuencia es que todos, de una u otra manera, tenemos algo de los «malvados» descriptos en los salmos. Pero el reconocimiento de esta realidad no tiene que dejarnos instalados en una situación de inmovilidad. El llamado del Salmo 32 es a salir de esa situación, porque es el modo de librarse de la angustia y la opresión interior. Dicho de otro modo, todos tenemos que preguntarnos si algunos de los sufrimientos que padecemos tienen que ver con haber equivocado el camino y la falta de capacidad o coraje para reconocerlo y poder decirlo.

También esta lectura e interpretación de Sal 32,10 abre a una perspectiva pastoral distinta frente a quienes son considerados como personas que se encuentran en el «mal camino». El hecho de que la apariencia muestre una cierta satisfacción en quien se encuentra en una posición de transgresión moral, sobre todo de orden público, no garantiza que realmente sea esa su vivencia más honda. Frente a esta realidad, un camino puede consistir en la intervención profética, advirtiendo al otro respecto de las posibles causas de su malestar. Otro camino puede ser el de la escucha, la cercanía, que señale, en el momento oportuno, las desventajas de encontrarse en el camino que no corresponde.

De esta manera, el v. 10 del Salmo 32 puede abrir una potencial vía de diálogo de los creyentes con quienes se encuentran en sendas alejadas de Dios, tomando en serio lo más conveniente para la condición humana. Esta vía de dialogo tiene que seguir el camino de la propia experiencia de debilidad y de caída. Es decir, quien puede reconocer en sí el dolor por haber equivocado el camino, quien ha experimentado el sufrimiento mientras se encontraba en dicha situación, está también en condiciones de ayudar a otros a reconocer su propio dolor y sufrimiento por encontrarse en el camino errado.

Por último, cabe acotar que la afirmación del salmo es que los sufrimientos del malvado son muchos. No se trata de algo irrelevante, pasajero, banal. La calificación cuantitativa tiene, en este caso, su valor suplementario. No se está hablando de la superficie de la experiencia humana. Se está abordando la cuestión de la felicidad o la no felicidad de la existencia toda. De ahí la relevancia del tema, la importancia de una clarificación sobre este punto y, eventualmente, una acción proporcionada a esta severa advertencia que nos deja la Palabra.

\author{
ÁNGel José Macín \\ Reconquista - Santa Fe \\ angel.macin@gmail.com
}

